\title{
ASYMPTOTIC ANALYSIS OF CHANNEL DIVISION MULTIPLE ACCESS SCHEMES FOR ULTRA-WIDEBAND SYSTEMS
}

\author{
Raul de Lacerda, Laura Cottatellucci, Aawatif Hayar
}

\author{
Institut Eurecom \\ Dpt. Communications Mobiles \\ 06904, Sophia Antipolis, France
}

\author{
Mérouane Debbah*
}

\author{
Supelec \\ Alcatel-Lucent Chair \\ 91190, Gif-sur-Yvette, France
}

\begin{abstract}
Channel Division Multiple Access (ChDMA) is a promising multiple access scheme for Ultra-Wide Band (UWB) systems based on the use of the Channel Impulse Responses (CIR) as user signatures. In this work, we modeled the UWB-CIR as linear combinations of continuous impulses of finite duration randomly delayed. Two different channels are considered: the first, very simplistic, generates multipaths that are uniformly distributed over the time; the second model, which introduces correlation between the delay and the energy of the paths, is built based on the channel power profile. The capacity is investigated assuming no Channel State Information (CSI) at the transmitters and perfect CSI at the receiver. As results, we derive the asymptotic capacity of the ChDMA scheme when the number of users and the number of frequency dimensions (which is proportional to the bandwidth) go to infinity with constant ratio. As a consequence, we observe that the asymptotic spectral efficiency depends only on the system load, the power delay profile, the noise variance and the pulse signal. The results are validated in certain conditions and compared with known results on CDMA systems.
\end{abstract}

\section{INTRODUCTION}

Ultra-WideBand (UWB) has been recently presented as a promising radio technology due to the large available bandwidth. UWB systems enables high data rates at short range as well as high temporal resolution with long Channel Impulse Responses (CIR) thanks to their large bandwidth, based on waveform radiations of "very short" pulses. Moreover, the system uses very low power spectral density, below the thermal noise of the conventional receivers, which is inherently difficult to be detected and does not cause significant interference to other systems. These appealing, fundamental properties of UWB radio system make it an ideal candidate for commercial, short-range, low power, low cost indoor communication systems such as Wireless Local Area Network (WLAN) and Personal Area Network (WPAN).

${ }^{*}$ This work was supported by Alcatel-Lucent within the Alcatel-Lucen Chair on flexible radio at SUPELEC.
Although a large amount of efforts has been devoted to develop efficient UWB systems during the last years, few studies focused on the design of efficient multiple access schemes able to benefit from low duty cycle transmissions. In 2006, a very simple technique called Channel Division Multiple Access (ChDMA) [1,2] was presented to exploit the duty cycle structure of the UWB signal and provide an interesting way to guarantee efficient multiuser communications in UWB systems. Benefiting from the fact that the coherence time is large (typically about $100 \mu \mathrm{s}$ ) in comparison with the delay spread (typically from 15-40 $\mathrm{ns}$, depending on the user environment), each user employs its impulse response to modulate its signal. The system shows many similarities with uplink code division multiple access (CDMA) systems with random spreading sequences, whose spectral efficiency is well known [3].

In this work, we analyze the asymptotic spectral efficiency of ChDMA systems under the assumption that the number of users and the size of the bandwidth increases at a constant ratio. To this aim, we modelled the UWB-CIR as linear combinations of continuous impulses of finite duration and employ two different power delay profiles: uniform and exponential profiles. During the evaluation of the system efficiency, we verify the impact of different system parameters.

\section{SYSTEM MODEL}

We consider an uplink UWB ChDMA system with $K$ single antenna users and a base station that employs a single receive antenna $[1,2]$. We also assume that users access simultaneously and synchronously (at symbol level). From the transmitter side, each user sends a low duty modulating signal with a symbol rate given by $f_{s}=T_{s}^{-1}$, where $T_{s}$ denotes the interval between consecutive symbol transmissions. The transmitted signal is distorted by the channel and the distortion can be regarded as a modulation. The CIR can be consider as a signature waveform, similarly to the spreading sequences of CDMA systems. At the receiver, the base station is able to detect the transmitted signals by using the CIR, which are specific to each user. This is the fundamental idea of the 
ChDMA. Without loss of generality, we assume $T_{s}$ equal to the maximum multipath delay (or delay spread) of the wireless environment, i.e. $T_{s}=T_{d}$, and equal for all the users. The system is impaired by additive white Gaussian noise.

The impulse response $h_{k}(t)$ of the channel between user $k$ and the base station can be written as

$$
h_{k}(t)=\sum_{\ell=1}^{L_{k}} \lambda_{k, \ell} g\left(t-\tau_{k, \ell}\right),
$$

where $L_{k}$ represents the number of distinguishable paths, $g(t)$ is the pulse signal employed in the signal transmission, $\lambda_{k, \ell}$ and $\tau_{k, \ell}$ are the amplitude and the delay of the $\ell$ th path of user $k$, respectively. The amplitudes $\lambda_{k, \ell}$ are random variables that follow a normal distribution with zero mean and variances $\sigma_{k, \ell}^{2}$ such that $\sum_{k=1}^{L_{k}} \sigma_{k, \ell}^{2}=P_{k}$. Without loss of generality, we assume that $P_{k}=1$. In fact, different values of $P_{k}$ can be taken into account by a multiplicative factor $a_{k}$ at the receiver that includes both, the effects of channel attenuation $P_{k}$ and the transmitted power $P_{t, k}$, i.e. $a_{k}=\sqrt{P_{k} P_{t, k}}$. Furthermore, we assume that all the users have the same number of distinguishable paths, i.e. $L_{k}=L$. As a consequence, the frequency response $H_{k}(f)$ of the channel between user $k$ and the base station can be written as

$$
H_{k}(f)=\sum_{\ell=1}^{L} \lambda_{k, \ell} G(f) \mathrm{e}^{-j 2 \pi f \tau_{\ell}},
$$

where $G(f)$ is the Fourier transform of the $g(t)$ function.

Then, in frequency domain, the signal received at the base station is given by

$$
\mathbf{y}=\mathbf{H A} \mathbf{s}+\mathbf{n},
$$

where $\mathbf{y}$ is the $N$-dimensional vector of received signal; $\mathbf{H}=$ $\left[\mathbf{h}_{1}, \ldots, \mathbf{h}_{K}\right]$ is an $N \times K$ matrix whose $k^{\text {th }}$ column $\mathbf{h}_{k}$ is the sampled frequency response of user $k ; \mathbf{A}$ is the $K \times K$ diagonal matrix of received amplitudes whose $k$-th diagonal element is $a_{k} ; \mathbf{s}$ is a $K$-dimensional vector of transmitted symbols (it is assumed to be i.i.d random variables with zero mean and unitary variance), and $\mathbf{n}$ is a vector that takes into account the additive white Gaussian noise with zero mean and variance $\sigma^{2}$. In order to keep the notation simple, we assume that $N$ is an even integer. The vector $\mathbf{y}$ is obtained sampling the received signal in the frequency domain at rate $\frac{1}{W_{c}}$, where $W_{c}$ represents the frequency resolution ${ }^{1}$. This implies that the baseband representation of the system is within the frequency interval $\left[-\frac{N}{2} W_{c}, \frac{N}{2} W_{c}\right]$.

\section{CASES OF STUDY}

Throughout this work we analyze the capacity of the above proposed multiple access scheme in the following two cases:

\footnotetext{
${ }^{1}$ In order to respect the coherence bandwidth of the system, the frequency resolution has to satisfy the following inequality $W_{c} \leq \frac{1}{T_{d}}$.
}

Case 1: The amplitudes $\lambda_{k, \ell}$ and the path delays $\tau_{k, \ell}$ are complex and real random variables, respectively. They are mutually independent and statistically independent over all the $K$ users and all the $L$ paths. The delays $\tau_{k, \ell}$ are random variables uniformly distributed in the interval $\left[0, T_{d}\right]$. The amplitudes $\lambda_{k, \ell}$ are random variables with zero mean and variances $\frac{1}{L}$. Furthermore, if $T_{d} \neq W_{c}^{-1}$, the number of multipath $L$ is assumed to be large enough that the random variable $H_{k}(f)$ is Gaussian with zero mean.

Case 2: The amplitudes $\lambda_{k, \ell}$ and the path delays $\tau_{k, \ell}$ of user $k$ are joint random variables with joint probability density function $f_{\Lambda_{1} \ldots \lambda_{L}, T_{1} \ldots T_{L}}\left(\lambda_{1}, \ldots \lambda_{L}, \tau_{1} \ldots \tau_{L}\right)$. They are independent over all users. The joint distribution $f_{\Lambda_{1} \ldots \lambda_{L}, T_{1} \ldots T_{L}}\left(\lambda_{1}, \ldots \lambda_{L}, \tau_{1} \ldots \tau_{L}\right)$ takes into account the power delay profile and the random variables $\lambda_{k \ell}$ are in general dependent of the delays $\tau_{k \ell}$. Also in this case, the marginal distributions of $\lambda_{k, \ell}$ are Gaussian with zero mean and variances $\sigma_{\Lambda_{\ell}}^{2}$ and the number of multipath $L$ is large enough that the random variable $H_{k}(f)$ is Gaussian with zero mean. The delays are random variables uniformly distributed in the interval $\left[\frac{(\ell-1) T_{d}}{L}, \frac{(\ell) T_{d}}{L}\right]$.

\subsection{Case 1}

Thanks to the independence of $\lambda_{k, \ell}$ and $\tau_{k, \ell}$ over all users, the columns of the matrix $\mathbf{H}$ are statistically independent. In order to characterize the matrix $\mathbf{H}$ completely, it is sufficient to determine the covariance matrix of one of the Gaussian vector $\mathbf{h}_{k}$, for $k=1, \ldots K$. The $m^{\text {th }}$ component of the vector $\mathbf{h}_{k}$ is

$$
h_{m, k}=\sum_{\ell=1}^{L} \lambda_{k, \ell} G\left(W_{c}(m-1)-\frac{W_{c} N}{2}\right) \mathrm{e}^{j 2 \pi W_{c}(m-1) \tau_{\ell}}
$$

with $m=1, \ldots, N$. Then, the $(m, n)$ element of the covariance matrix $\mathbf{C}^{(1)}=\mathbb{E}\left\{\mathbf{h}_{k} \mathbf{h}_{k}^{H}\right\}$ is

$$
\begin{aligned}
C_{m, n}^{(1)}= & \sum_{u=1}^{L} \sum_{v=1}^{L} \mathbb{E}\left\{\lambda_{k, u} G\left(W_{c}(m-1)-\frac{W_{c} N}{2}\right)\right. \\
& \cdot \mathrm{e}^{j 2 \pi W_{c}(m-1) \tau_{u}} \lambda_{k, v}^{*} G^{*}\left(W_{c}(n-1)-\frac{W_{c} N}{2}\right) \\
& \left.\cdot \mathrm{e}^{-j 2 \pi W_{c}(n-1) \tau_{v}}\right\} \\
= & G\left(W_{c}(m-1)-\frac{W_{c} N}{2}\right) G^{*}\left(W_{c}(n-1)-\frac{W_{c} N}{2}\right) \\
& \cdot \sum_{u=1}^{L} \mathbb{E}\left\{\lambda_{k, u} \lambda_{k, u}^{*}\right\} \mathbb{E}\left\{\mathrm{e}^{j 2 \pi W_{c}(m-n) \tau_{u}}\right\} \\
= & G\left(W_{c}(m-1)-\frac{W_{c} N}{2}\right) G^{*}\left(W_{c}(n-1)-\frac{W_{c} N}{2}\right) \\
& \cdot\left(\frac{1}{T_{d}} \int_{0}^{T_{d}} \mathrm{e}^{j 2 \pi W_{c}(m-n) \tau} d \tau\right) \sum_{u=1}^{L} \sigma_{\Lambda_{u}}^{2}
\end{aligned}
$$




$$
\begin{aligned}
= & G\left(W_{c}(m-1)-\frac{W_{c} N}{2}\right) G^{*}\left(W_{c}(n-1)-\frac{W_{c} N}{2}\right) \\
& \cdot \mathrm{e}^{j \pi W_{c}(m-n) T_{d}} \operatorname{sinc}\left(W_{c}(m-n) T_{d}\right) .
\end{aligned}
$$

For simplification purposes, let $g(t)$ be a Dirac impulse, i.e. $g(t)=\delta(t)$. Under this assumption, we have that

$$
C_{m, n}^{(1)}=\mathrm{e}^{j \pi W_{c}(m-n) T_{d}} \operatorname{sinc}\left(W_{c}(m-n) T_{d}\right) .
$$

Therefore, the matrix $\mathbf{C}$ for case 1 can be written as

$$
\mathbf{C}^{(1)}=\mathbf{S}\left(W_{c} T_{d}, W_{c} T_{d}\right),
$$

where $\mathbf{S}(x, y)$ is a $N \times N$ matrix with elements given by

$$
s_{i, j}(x, y)=\operatorname{sinc}(x(i-j)) \mathrm{e}^{j \pi y(i-j)} .
$$

Then, thanks to the Toeplitz structure of the matrix $\mathbf{C}^{(1)}$, the following eigen-decomposition holds when $N \rightarrow \infty$ [4],

$$
\mathbf{C}_{\infty}^{(1)}=\lim _{N \rightarrow \infty} \mathbf{F}_{N}^{H} \mathbf{D}^{(1)} \mathbf{F}_{N}
$$

being $\mathbf{F}_{N}$ the Fourier matrix and $\mathbf{D}^{(1)}$ a diagonal matrix with elements

$$
d_{n, n}= \begin{cases}\frac{1}{W_{c} T_{d}} & , \text { for } 0 \leq n-1 \leq N W_{c} T_{d} \\ 0 & , \text { otherwise. }\end{cases}
$$

As one can observe, the obtained $\mathbf{D}^{(1)}$ does not depend on $L$, but only on the delay spread $T_{d}$ and the frequency resolution $W_{c}$

\subsection{Case 2}

We assume that the elements of one set $\left(\lambda_{k, j}, \tau_{k, j}\right)$ are statistically dependent of each other but statistically independent of any element of any other set $\left(\lambda_{k^{\prime}, j^{\prime}}, \tau_{k^{\prime}, j^{\prime}}\right) \forall k^{\prime} \neq k$ and $j^{\prime} \neq j$, i.e.,

$$
f\left(\lambda_{k, 1}, \ldots, \lambda_{k, L}, \tau_{k, 1}, \ldots, \tau_{k, L}\right)=\prod_{\ell=1}^{L} f_{\ell}\left(\lambda_{k, \ell}, \ldots, \tau_{k, \ell}\right)
$$

Furthermore, consistently with the general assumptions in Section 2 the marginal distribution of $\tau_{k, \ell}$ is uniformly distributed in the interval $\left[\frac{T_{d}(\ell-1)}{L}, \frac{T_{d} \ell}{L}\right], \mathbb{E}\left\{\lambda_{k, \ell}\right\}=0$, and $\mathbb{E}\left\{\left|\lambda_{k, \ell}\right|^{2}\right\}=$ $\sigma_{\Lambda_{\ell}}^{2}$ with $\sum_{k=1}^{L_{k}} \sigma_{\Lambda_{\ell}}^{2}=1$. The $(m, n)$-th element of the covariance matrix $\mathbf{C}^{(2)}=\mathbb{E}\left\{\mathbf{h}_{k} \mathbf{h}_{k}^{H}\right\}$ is given by

$$
\begin{aligned}
C_{m, n}^{(2)}= & \sum_{\ell=1}^{L} \sigma_{\Lambda_{\ell}}^{2} G\left(W_{c}(m-1)-\frac{W_{c} N}{2}\right) \\
& \cdot G^{*}\left(W_{c}(n-1)-\frac{W_{c} N}{2}\right) \\
& \cdot \mathbb{E}\left\{\mathrm{e}^{j 2 \pi W_{c}(m-n) \tau_{\ell}}\right\}
\end{aligned}
$$

$$
\begin{aligned}
= & \sum_{\ell=1}^{L} \sigma_{\Lambda_{l}}^{2} G\left(W_{c}(m-1)-\frac{W_{c} N}{2}\right) \\
& \cdot G^{*}\left(W_{c}(n-1)-\frac{W_{c} N}{2}\right) \mathrm{e}^{j \pi W_{c}(m-n) \frac{T_{d}}{L}(2 \ell-1)} \\
& \cdot \operatorname{sinc}\left(\frac{W_{c} T_{d}}{L}(m-n)\right)
\end{aligned}
$$

By assuming that $g(t)$ is a Dirac, i.e. $g(t)=\delta(t)$, (14) reduces to

$$
\begin{aligned}
C_{m, n}^{(2)}= & \sum_{\ell=1}^{L} \sigma_{\Lambda_{\ell}}^{2} \cdot \mathrm{e}^{j \pi W_{c}(m-n) \frac{T_{d}}{L}(2 \ell-1)} \\
& \cdot \operatorname{sinc}\left(\frac{W_{c} T_{d}}{L}(m-n)\right) .
\end{aligned}
$$

Therefore, the matrix $\mathbf{C}$ for case 2 can be written as

$$
\mathbf{C}^{(2)}=\sum_{\ell=1}^{L} \sigma_{\Lambda_{\ell}}^{2} \cdot \mathbf{S}\left(\frac{W_{c} T_{d}}{L}, \frac{W_{c} T_{d}}{L}(2 \ell-1)\right) .
$$

Asymptotically, for $N \rightarrow \infty$, the following eigen-decomposition holds

$$
\mathbf{C}_{\infty}^{(2)}=\lim _{N \rightarrow \infty} \mathbf{F}_{N}^{H} \mathbf{D}^{(2)} \mathbf{F}_{N}
$$

with

$$
d_{n, n}= \begin{cases}\frac{\sigma_{\Lambda_{\ell}}^{2} L}{W_{c} T_{d}} & \text { for } \frac{N W_{c} T_{d}}{L}(\ell-1) \leq n-1 \leq \frac{N W_{c} T_{d}}{L} \ell, \\ 0 & \text { otherwise. }\end{cases}
$$

As one can observe, we have that $\mathbf{D}^{(2)}=\mathbf{D}^{(1)}$ for the case where $\lambda_{k, \ell}$ have identical distributions with the same variances, i.e., $\sigma_{\Lambda_{\ell}}^{2}=\frac{1}{L}, \forall \ell=1, \ldots, L$. This result shows that the assumption that each time delay delay belongs to a fixed interval disjoint from the others does not introduce any modification/constraint on the asymptotic result if the power delay profile is constant compared to case 1 .

\section{SPECTRAL EFFICIENCY}

The spectral efficiency analysis of the ChDMA system is similar to the one presented by Verdu and Shamai [3] for CDMA systems with random spreading codes. The spectral efficiency per frequency bin is given by

$$
\begin{aligned}
\gamma & =\frac{1}{N} \log _{2} \operatorname{det}\left(\mathbf{I}_{N}+\rho \mathbf{H} \mathbf{A} \mathbf{A}^{H} \mathbf{H}^{H}\right) \\
& =\frac{1}{N} \log \operatorname{det}\left(\mathbf{I}_{N}+\rho \sqrt{\mathbf{D}^{(a)}} \mathbf{V} \mathbf{A} \mathbf{A}^{H} \mathbf{V}^{H}{\sqrt{\mathbf{D}^{(a)}}}^{H}\right)
\end{aligned}
$$


where $\rho=\frac{\gamma}{\beta} \frac{E_{b}}{N_{0}}, a=1,2$ if the channel is modelled as in case 1 or in case 2 respectively, and $\mathbf{V}$ is an $N \times K$ matrix with random i.i.d. entries having zero mean and unitary variance.

The following theorem provides the spectral efficiency per frequency bin as $K, N \rightarrow \infty$ with constant ratio. In asymptotic condition this figure becomes deterministic and depends on few macroscopic system parameters.

Theorem 1. Let $\mathbf{H}$ be and $N \times K$ matrix of random independent columns and let the elements of each column be correlated Gaussian with zero mean and covariance matrix $\frac{\mathbf{C}}{N}$. Let us assume that asymptotically as $N, K \rightarrow \infty$, the empirical eigenvalue distributions of the matrices $\mathbf{C}$ and $\mathbf{A A}^{H}$ converge to the deterministic distribution functions $F_{\mathbf{C}}(v)$ and $F_{|\mathbf{A}|^{2}}(p)$, respectively. Then, when $K, N \rightarrow \infty$ with $\frac{K}{N} \rightarrow \beta$, the spectral efficiency $\gamma$ converge to a deterministic limit

$$
\gamma_{\infty}=\frac{\beta}{\ln (2)} \int_{0}^{\rho} \mathrm{d} z \int_{0}^{+\infty} \frac{p \eta(z) \mathrm{d} F_{|\mathbf{A}|^{2}}(p)}{1+p z \eta(z)}
$$

with

$$
\eta(z)=\int \eta(v, z) \mathrm{d} F_{\mathbf{C}}(v)
$$

and

$$
\frac{1}{\eta(v, z)}=\frac{1}{v}+\beta \int \frac{p F_{|\mathbf{A}|^{2}}(p)}{\frac{1}{z}+p \int \eta(v, z) \mathrm{d} F_{\mathbf{C}}(v)}
$$

being $\eta(v, z)$ the unique positive solution to $(23)^{2}$.

For case 1 (see Eq. (11)) and case 2 (see Eq. (18)) the limiting eigenvalue probability density functions of the covariance matrix $\mathbf{C}$ are given by

$$
f_{\mathbf{C}}^{(1)}(v)=\left(1-W_{c} T_{d}\right) \delta(v)+W_{c} T_{d} \delta\left(v-\frac{1}{W_{c} T_{d}}\right)
$$

and

$$
f_{\mathbf{C}}^{(2)}(v)=\left(1-W_{c} T_{d}\right) \delta(v)+\frac{W_{c} T_{d}}{L} \sum_{\ell=1}^{L} \delta\left(v-\frac{\sigma_{\Lambda_{\ell}}^{2} L}{W_{c} T_{d}}\right)
$$

respectively.

Under the assumption that all the received powers are equal to one the expressions (21), (22) and (23) simplify considerably and depends only on $T_{d}, W_{c}$ and $\beta$.

\footnotetext{
${ }^{2}$ Due to the lack of space, the proof of this theorem could not be included.
}

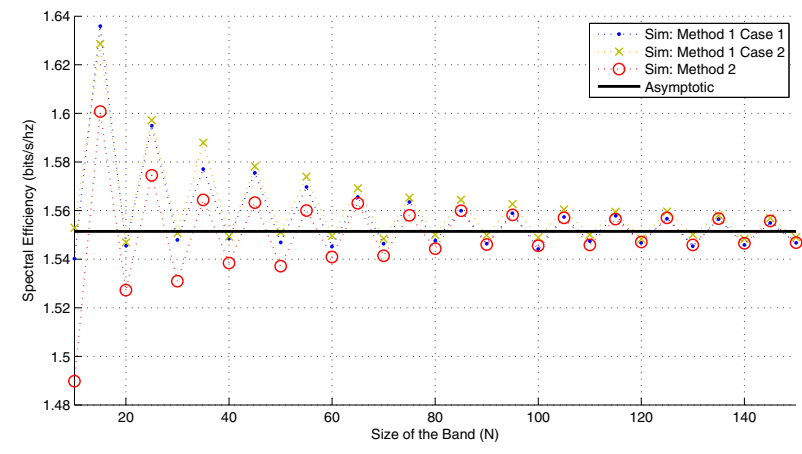

Fig. 1. Impact of the number of frequency samples $N$.

\section{RESULTS}

In this section, we present the results obtained by the asymptotic analysis (Eq. 21) and compare them with the results obtained by Monte Carlo simulations of ChDMA systems of finite dimensions (Eq. 19). For finite simulated systems, we consider two different methods to build the channel matrix: the first one, labelled Method 1, creates the channel by generating random multipaths under the restrictions discussed in Section 3. The second method, referred to as Method 2, is based on the random generation of the channel by employing the diagonal matrix $\mathbf{D}^{(a)}$. In fact, this is a fundamental step to derive the asymptotic equation.

The results are obtained averaging over 500 Monte Carlo simulations. We considered an environment with a maximum delay spread of $25 \mathrm{~ns}$. Furthermore, when $\beta$, the number of frequency samples $N$, the frequency resolution $W_{c}$, the ratio $E_{b} / N_{0}$ or the number of multipaths $L$ are not given, we assume by default that they are respectively $0.8,50,40 \mathrm{MHz}$, $5 \mathrm{~dB}$ and 100 . Similarly, by default the received power distribution is $F_{|\mathbf{A}|^{2}}(p)=1$ for $p \leq 1$, i.e. we assume perfect power control.

\subsection{Validation}

As a first step, we validate numerically the assumptions made to derive the asymptotic results and we compare the resulting spectral efficiency with known results in the literature.

In Fig. 1, the impact of the number of frequency dimensions on the spectral efficiency is presented. As the system dimensions increase, the spectral efficiency of all the simulated channels converge to the asymptotic theoretical value. Convergence was also observed for different system configurations, but the speed of converge is intimately dependent of all the system parameters.

It is possible to verify that the spectral efficiency $\gamma_{i} n f t y$ reduces to the expression of the asymptotic spectral efficiency per chip of CDMA systems with random spreading codes 


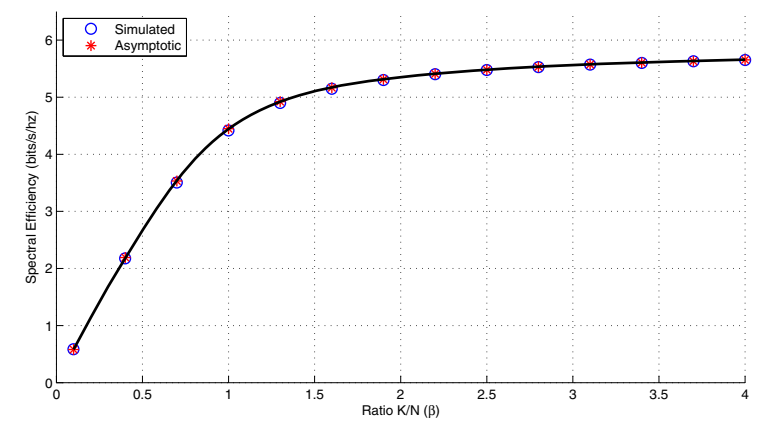

Fig. 2. Validation with the CDMA result $\left(\frac{E_{b}}{N_{0}}=10\right)$.

studied by Verdú and Shamai in [3], when $T_{d}=W_{c}^{-1}$ and the power delay profile is uniform. Fig. 2 compares the spectral efficiency as a function of the system load $\beta$ in case of finite simulated channels with the asymptotic theoretical spectral efficnecy of CDMA as $\frac{E_{b}}{N_{0}}=10 \mathrm{~dB}$. Although the plots are obtained assuming a very low number of frequency dimensions in the finite case $(N=50)$, the two curves match completely for different values of $\beta$.

\subsection{Power Delay Profile}

In this section we investigate the impact of a nonuniform power delay profile (PDP) on the spectral efficiency of ChDMA systems. We consider an exponentially decaying PDP when the assumptions of case 2 are enforced. This type of profile is more realistic and it is largely used to characterize the UWB environment [5,6]. Such profile is defined by assuming $\sigma_{\Lambda_{\ell}}^{2}=$ $\frac{\alpha_{1}}{L\left(1-e^{\left.-\alpha T_{d}\right)}\right.} e^{-\alpha \tau_{\ell}}$, where $\alpha$ is the decay factor.

In order to analyze the impact of the PDP, we consider three different decay factor values of the exponential decaying profile. Fig. 3 shows the spectral efficiency of a ChDMA system with exponential PDP as a function of the load $\beta$ for $\alpha=0.01,1,5,10$. Furthermore, the performance of the system with exponentially decaying PDP is compared to case of uniform PDP (solid line) with $\alpha=0$. At the parameter $\alpha$ increases the spectral efficiency increases if the system load $\beta$ is sufficiently large. This is due to the concentration of the channel energy in a small portion of the channel interval. This concentration determines an inefficient utilization of the available system dimensions. Then, the receiver cannot separate completely the information transmitted by different users. On the other hand, for lower values of $\alpha$, the system performance tends to the performance of a system with uniform PDP, as we could expect.

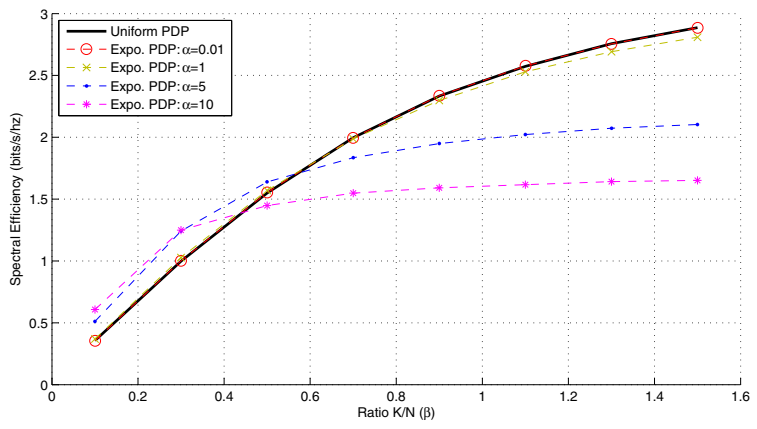

Fig. 3. Impact of the Power Delay Profile.

\section{CONCLUSIONS}

In this work we provided an analysis of ChDMA systems in terms of spectral efficiency as the system size grows large. Under this asymptotic conditions the spectral efficiency is independent of the channel realization and becomes deterministic. Two channel models were considered and analytic expressions of the spectral efficiency were derived based on results of random matrix theory. We validated the asymptotic equations via simulation results and verified the impact of the power delay profile on ChDMA systems.

As perspectives, we would like to tackle problems related to errors on channel estimations, the impact of training on the spectral efficiency of the system and refine the UWB channel models to include typical behaviours such as the non-linear frequency attenuation.

\section{REFERENCES}

[1] R. L. de Lacerda Neto, M. Debbah, and A. Menouni Hayar, "Channel division multiple access," in 1st IEEE International Conference on Wireless Broadband and Ultra-Wideband Communications, New South Wales, Australia, Mar. 2006.

[2] R. L. de Lacerda Neto, A. Menouni Hayar, and M. Debbah, "Channel division multiple access based on high UWB channel temporal resolution," in 64th IEEE Vehicular Technology Conference 2006 Fall, Montreal, Canada, Sept. 2006.

[3] S. Verdú and S. Shamai, "Spectral efficiency of CDMA with random spreading," IEEE Transactions on Information Theory, vol. 45, pp. 622-640, Mar. 1999.

[4] R. M. Gray, Toeplitz and Circulant Matrices: A Review, Technical Rept. No. 6504-1, Inform. Sys. Lab., Stanford Univ., Stanford, CA, April 1977.

[5] A. Saleh and R. Valenzuela, "A statistical model for indoor multipath propagation," Selected Areas in Communications, IEEE Journal on, vol. 5, no. 2, pp. 128-137, 1987.

[6] D. Cassioli, M. Z. Win, and A. F. Molisch, "The ultra-wide bandwidth indoor channel from statistical model to simulations," IEEE Journal on Selected Areas in Communications, vol. 20, no. 6, pp. 1247-1257, Aug. 2002. 